\section{Examining the relationship between traditional Chinese medicine and conventional medicine in breast cancer patients medicated with estrogen antagonists}

\section{Jill Hervik, 1 Odd Mjåland2}

1Pain Clinic, Vestfold Hospital, Tonsberg; 2Department of Abdominal Surgery, Sorlandet Hospital, Kristiansand, Norway

\begin{abstract}
Breast cancer is the most common type of cancer among women, with a $10.4 \%$ incidence rate worldwide. It is the fifth most common cause of death. Treatment includes surgery, radiation and drugs. Conventional treatment of estrogen receptor positive types of tumors involves the use of hormone therapy. The sideeffects of these drugs, i.e. menopausal type symptoms, are an extra burden to women with breast cancer. Further use of drugs to treat such side-effects often provokes adverse symptoms, prompting a search for alternative types of treatment. This paper aims to review research into the use of traditional Chinese medical acupuncture to combat side-effects in breast cancer patients medicated with estrogen antagonists, and examine its complementary role.
\end{abstract}

\section{Introduction}

Breast cancer is the most common type of cancer among women; 1.38 million new cancer cases were diagnosed in 2008.1 Incidence rates are lower in developing countries; the incidence of breast cancer has been much lower in the East compared to the West. The growth of developing countries and adoption of western lifestyles and culture seems to bring with it a change in disease patterns, including breast cancer. Demographic trends indicate that even more women will face breast cancer in the future. According to the World Health Organization (WHO), in 2008 incidence rates per 100,000 women varied from 19.3 in Eastern Africa to 89.9 in Western Europe. Breast cancer ranks as the fifth cause of death worldwide with 458,000 deaths, and was the most frequent cause of death in women in both developing and developed regions. ${ }^{2}$

Treatment of breast cancer involves surgery, radiation and drugs (chemotherapy and endocrine). Three types of receptors may or may not be present in breast cancer cells. These are: estrogen, progesterone and HER 2. This paper aims to examine the role of traditional Chinese acupuncture (TCA) in women with estrogen receptor positive (ER+) breast cancer, suffering from side-effects of estrogen antagonist medication. In accordance with European guidelines, ${ }^{3}$ medication with estrogen antagonists is recommended for a minimum of five years for women with this type of tumor. In Norway, approximately $55 \%$ of breast cancers are estrogen receptor positive and, therefore, adjuvant hormonal therapy is appropriate. $^{4}$ For the last 25-30 years, tamoxifen (TAM) has been used in ER+ breast cancers and has been shown to reduce the incidence of recurrence and death by $47 \%$ and $26 \%$, respectively. ${ }^{5}$ Recently, aromatase inhibitors have been incorporated into standard care in ER+ breast cancers in postmenopausal women, including natural menopause and chemically induced menopause. Post-menopausal women produce most of their estrogen in the adrenal gland, converted from androgens, and in fatty tissue. Aromatase inhibitors (AIs) block the synthesis of testosterone to estradiol by inhibiting the aromatase enzyme. In premenopausal women, most of their estrogen is produced in the ovaries. TAM works by binding itself to estrogen receptors in the breast, blocking estrogen from binding and, therefore, deactivating the receptor. TAM has traditionally been used primarily in both pre- and postmenopausal women, with a switch to an AI when the postmenopausal state is confirmed. Recently, several controlled studies indicate the use of aromatase inhibitors as the initial drug choice in postmenopausal women.6,7 Disease recurrence in other parts of the body in breast cancer patients has been shown to peak after two years. ${ }^{5}$ AIs have demonstrated superiority to TAM in the reduction of early recurrence and improved long-term survival, as seen in two well known trials: the ATAC, anastrozole alone or in combination with TAM, and the IES, exemestane after 2-3 years of TAM, trials. The latter compared the sideeffects of TAM with AIs, showing a greater tolerance for AIs compared to TAM. 8

Traditional Chinese Medicine (TCM) consists of herbal medicine and acupuncture. Treatments are usually individualized according to diagnosis of imbalance and patterns of disharmony. Diagnostic procedures consist of the four examinations: looking, listening, asking and touching. While Western medicine tries to find a specific cause of a disease or sickness by narrowing and condensing information to isolate and then treat, TCM takes a wider look at disease, assessing the whole individual, physiologically and psychologically by logically assessing various systems, including yin, yang, qi, blood and body fluids. The end result is an organization of signs and symp-
Correspondence: Jill Hervik, Pain Clinic, Vestfold Hospital, Tønsberg, Norway.

E-mail: jill.hervik@siv.no

Key words: traditional Chinese medicine, breast cancer, estrogen antagonist treatment, sideeffects.

Received for publication: 23 March 2012 Accepted for publication: 18 May 2012.

This work is licensed under a Creative Commons Attribution NonCommercial 3.0 License (CC BYNC 3.0).

(C) Copyright J. Hervik and O. Mjåland, 2012 Licensee PAGEPress, Italy

Alternative Medicine Studies 2012; 2:e11

doi:10.4081/ams.2012.e11

toms, discerning relationships between body processes. Therapy attempts to restore balance to the individual. TCM has been passed down for centuries in ancient texts. The history of these texts concerning women's medicine is described by Maciocia. ${ }^{9}$ The ancient texts are a basis for critical evaluation, development, clinical observation and research. According to Maciocia, during the Han dynasty (206 BC-AD 220), a gynecologist was called a breast doctor or a women's doctor. Books were written on gynecology even in the period before the Han dynasty. The Discussion of Prescriptions of the Golden Chest, originally written in AD 220 by Zhang Zhong Jing, 10 describes herbal treatments for pregnancy, post-partum diseases and a miscellany of women's diseases, and was the basis for subsequent literature on the subject of gynecology. The description of different types of breast tumors, including their physiology and severity, is found in early Chinese medical texts. Over 100 names for tumors can be found in this medical literature; the most frequently mentioned was rock 12 . The first clinical description of breast cancer can be found in the Yellow Emperors Classic of Internal Medicine from 100 BC. 11 The description indicated a prognosis of ten years from the time of diagnosis, including disease progression, metastasis and death. Breast tumors were called $\mathrm{Ru} \mathrm{Pi}$, which indicated that the lump was benign, or Ru Yan, which indicated a more serious, malignant tumor. A description of these tumors can be found in the Classic of the Central Treasure (Han dynasty 206 BC220AD). ${ }^{12}$ During the late Qing dynasty (16441911), moves were made to integrate Western medicine with Chinese medicine, although full integration was not achieved until around 1950. Today, the trend in China is still to integrate Eastern and Western medicine. Literature seems to suggest that most women diagnosed with breast cancer choose surgery and adjuvant therapies while TCM is used to 
control side-effects and toxicities with the aim of improving quality of life and preventing recurrence. 13

\section{Methods and search strategy}

Medline, Alternative Health Watch (EBSCO), CINAH and Cochrane Central databases were searched for articles published between 1985 and December 2010. Only articles originally published in English or translated to English were considered. We combined the words breast cancer with the following search strings: estrogen antagonists or antiestrogens or TAM or aromatase inhibitors or anastrozole and side-effects or acupuncture or traditional Chinese medicine or adjuvant treatment. Reference lists from individual trials and reviews were also searched for pertinent papers.

\section{Conventional treatment of breast cancer and traditional Chinese medicine}

Breast cancer is usually treated with surgery. Conventional medicine classifies tumors in stages from 1 to 4 by size, whether or not there is lymph node involvement or whether the tumor has metastasized. Stage 1 cancers are often treated with a lumpectomy and radiation. Stage 2 and 3 cancers have a progressively poorer prognosis due to risk of relapse; they are usually treated with a lumpectomy or mastectomy, with possible lymph node removal and chemotherapy. Stage 4, metastatic cancer, has spread to distant areas of the body and has a poor prognosis. It is managed by various combinations treatments including surgery, radiation, chemotherapy and other targeted therapies.

TCM considers cancer to be due to congealed phlegm, blood stasis and toxicity. Stagnation due to physical, mental or environmental factors hinders the elimination of toxic waste. With a reduction in the efficacy of the immune system causing stagnation, cell function is stressed and tumors can arise. Surgery from a TCM perspective results in damaging qi and blood, causing qi deficiency and blood stasis. The function of the spleen in containing qi and blood is affected. Anesthesia disturbs the heart and the functions of hun (ethereal soul) and po (corporeal soul) disrupting qi and blood, causing disturbance of shen. Qi and yin become deficient, resulting in symptoms such as nausea, pain, bruising and stiffness of the operated area.

Radiation causes cell death that is not selec- tive to cancer cells but has an impact on the whole radiated area; an inflammatory reaction causes a local burn and systemic fatigue. This external exposure to heat damages spleen qi and dries out fluids, and produces local heat and stagnation. The transforming and transporting function of the spleen is affected, heat and fire in the upper part of the body present as agitation and often insomnia, while deficiency in the form of damp heat is often seen in the lower part of the body. 14

Chemotherapy is usually given for stage $2-4$ cancers and is particularly beneficial in estrogen receptor-negative (ER-) disease. A trial by the Early Breast Cancer Trialists Collaberative group reported that adjuvant chemotherapy produced significant reductions in breast cancer recurrence by $35 \%$ in women under 50 , and by $20 \%$ for women between 50 and 69 years of age. ${ }^{5}$ Patients receive treatments in sessions, usually for 3-6 months the aim being to destroy fast growing or dividing cancer cells. The treatment does not, however, only destroy cancer cells but also fast-growing normal cells, potentially causing serious side-effects. The most common regimes are andriamycin with cytoxan, cytoxan methotrexate with 5 flourauracil, and taxol or taxotere which cause erosion of mucous membranes, resulting in dry mouth, bleeding gums and sores in the mouth; vomiting, abdominal pain, diarrhea, skin rashes and palpitations are also sometimes experienced. More serious reactions such as esophagitis, pancreatitis and bleeding ulcers are also known side-effects. Approximately a week after treatment women often complain of fatigue, dizziness, insomnia, fever, joint pains, cognitive fogginess, hot and cold sweats, palpitations and symptoms of anemia. Occasionally, problems of systemic toxicity occur causing organ dysfunction and potential failure. All body systems are depleted, including yin, yang, blood and body essences. Cytotoxic activity affects the organs and bone marrow, causing general deficiency. Kidney jing is affected, consequently the production of bone marrow fails, and in turn bones and joints are not nourished, the central nervous system is affected and blood is depleted. Often symptoms linger even when the body begins its recovery phase, usually from around the $10^{\text {th }}$ to the $12^{\text {th }}$ day after treatment. However, new symptoms may appear such as hair loss. Hair is nourished by blood, blood deficiency leads to alopecia and is an indirect indication of the function of the kidneys. Many women also complain of continuous bad memory and mental cloudiness, also an indication of kidney xu. There is some concern that these symptoms can become chronic and this is an area of current research interest in Western medicine.15,16 Nausea and vomiting is one of the most severe side-effects of chemotherapy with $30-40 \%$ of women experiencing nausea up to one week after receiving treatment.17,18 Chemotherapy and anesthesia induced nausea is an indication for acupuncture that in general has been recognized in Western medicine. Nausea is an indication recommended by WHO for acupuncture treatment. Queen's University, Belfast has produced numerous well controlled studies on nausea showing a treatment benefit in $60-70 \%$ of participants, compared to $30 \%$ receiving sham, when acupuncture is applied to P6 (Neiguan). ${ }^{14}$ Acupuncture was shown to be slightly more effective than ondansetron (zofran) for the treatment of post-operative nausea in an American study of 40 women undergoing breast surgery. ${ }^{19}$ Another serious side-effect of chemotherapy is leukopenia or decreased white blood cell count, lowering immunity. In a review and analysis of reports on controlled clinical acupuncture trials in 2002, the WHO suggested acupuncture as a suitable treatment for leukopenia. One of the studies they reviewed indicated that acupuncture was more effective than 2 leading drug treatments for chemotherapy induced leukopenia. ${ }^{20}$

Western medicine often starts to treat a disease when it presents symptoms. Treatment of breast cancer is largely cytotoxic, undermining physiological functions, treating the clinical manifestations but causing new symptoms. Though regrettable, this is essential to ensure patient survival. TCM can be useful for not only treating the side-effects of conventional medicine and supporting the patient's bodily functions, so that Western treatment can be as effective as possible, but it can also be used as part of prevention strategies in women with breast cysts and those genetically disposed to breast cancer. Research suggests that women who have had breast cysts are more likely to present with breast cancer at a later date than women who have not. ${ }^{21}$ To our knowledge, no studies have been conducted in the field of prevention. Blood stasis and or congealed phlegm are considered to be involved in most cancerous tumors. TCM doctors look for signs of abnormal microcirculation, indicating a possible pre-cancerous state. Research studies in China have found that cancer patients have high fibrinogen levels in the blood; tumors are often surrounded by fibrin preventing the entrance of immune cells and chemotherapy. ${ }^{22}$

\section{Side-effects of estrogen antag- onists}

A study of 452 breast cancer patients compared the side effects of aromatase inhibitors to TAM from the patient's perspective. ${ }^{23}$ The most bothersome side-effects experienced by the 452 women were hot flashes, weight gain, insomnia and joint aches. Other side-effects 
the women complained of were loss of libido, mood changes, muscle aches, vaginal dryness and vaginal discharge (Table 1). Severe osteoporosis was seen in $2.9 \%$ of TAM users and $5.7 \%$ of AI users. Results appear to be similar in both drug types apart from joint aches that was a problem for nearly twice as many women taking AIs (this has also been demonstrate in other similar studies).24,25 Although participants reported that hot flashes, weight gain, insomnia and joint aches were the most bothersome, $70-84 \%$ of them reported problems with loss of libido, mood changes, muscle aches and vaginal symptoms, collectively contributing to a reduced quality of life. A survey investigating side-effects of aromatase inhibitors carried out by the Breast Cancer Action group found that $97.7 \%$ of the 1,199 respondents had experienced one or more side-effects. ${ }^{15}$ Over $50 \%$ reported problems with hot flashes and symptoms including bone pain, tiredness, muscle pain, insomnia, weight gain, mental fuzziness, increased sweating and anxiety. Hair thinning and depression were reported by more than $30 \%$ of the women. Non-compliance with drug treatment was recorded in 333 (27\%) women. Among respondents who reported having stopped taking AIs because of experiencing two or more sideeffects, $76 \%$ named joint/bone/muscle pain to be the main reason. International guidelines suggest that estrogen antagonists should be used for a minimum of five years. These women are not on a short course of drug therapy that they can endure for a limited period. The consequences of hormonal therapy are long term, affecting all aspects of daily life, psychologically and physically, creating a need to treat side-effects. Discontinuation of antiestrogen medication due to intolerable sideeffects can have dire consequences.

\section{Hot flashes}

Hot flashes and sweating are reported as the most prominent side-effect of TAM and AI medication. ${ }^{26-28}$ These episodes may be accompanied by other physical reactions including sweating, facial redness, chills, palpitations, dizziness, nausea, paresthesia and acute shortness of breath. Emotional symptoms include anxiety, panic and emotional incontinence. ${ }^{29} \mathrm{~A}$ combination of hot flashes, sleep disturbances and fatigue is often seen, and these symptoms seem to directly affect each other, ${ }^{30}$ reducing quality of life. Hot flashes are defined as recurrent transient periods of flushing, sweating and a sensation of heat, often accompanied by palpitations and a feeling of anxiety, sometimes followed by chills and $a$ sensation of heat that is associated with objective signs of cutaneous vasodilatation and $a$ subsequent drop in core temperature, ${ }^{31}$ which may be accompanied by sweating, flushing, palpitations, anxiety, irritability and even panic. ${ }^{32}$ Hot flashes are frequently associated with menopause and ovarian failure and are, therefore, assumed to be caused by low sex hormone levels. The exact mechanisms of hot flashes are not known. However, several theories have been postulated. It has been suggested that dysfunction of the hypothalamic thermoregulatory processes is due to lower estrogen levels, affecting plasma levels of several neurotransmitters. ${ }^{33}$ This, in turn, causes the body thermostat to function at a lower level and with reduced range. Neurotransmitters including norepinephrine and serotonin are considered to be the main neurotransmitters involved in temperature regulation, dysfunction leads to hot flashes and sweating due to vasodilatation. $\beta$-endorphins, which are stimulated by estrogen metabolites, inhibit the production of norepinephrine in the hypothalamus, maintaining a homeostatic body temperature. A rise in norepinephrine due to lower estrogen levels will, in turn, cause a rise in core temperature. 34,35

For healthy women in the West, hormone replacement therapy (HRT) is an option for treating hot flashes. Historically, menopausal symptoms, especially hot flashes and night sweats, have not been common experiences for women in the East and, consequently HRT has not been widely used. Women with breast cancer do not have the option of using HRT. Indeed, it is contraindicated in ER+ breast cancer because of the potential increased risk of recurrence. Other pharmacological methods of treatment include antihypertensive clonidine, steroids, anti-depressants and gabapentin that often have adverse effects, such as hypotension, weight gain, nausea and cognitive problems, respectively. ${ }^{36}$ The use of selected serotonin inhibitors (SSRIs), anti-depressives used to treat both hot flashes and depression in women with breast cancer has recently been the focus of some controversy. In the USA, anti-depressants are the most popular type of treatment for vasomotor instability. 37 TAM is biologically transformed into the powerful anti-estrogen endoxifen by the cytochrome P450 pathway. SSRIs can inhibit this pathway and, therefore, TAM's effectiveness in breast cancer patients taking both medicines. ${ }^{38}$ Also, the efficacy and toxicity of TAM varies widely among individuals, with up to $35 \%$ of women with advanced ER+ breast cancer not responding to TAM. ${ }^{39}$ The substantial side-effects of Western drugs used to treat hot flashes in women medicated with antiestrogens indicate that there is a significant role for acupuncture, which when compared with the anti-depressive venlafaxine (effexor) in a study carried out in Detroit $(n=50)$, proved not only to be as effective in reducing hot flashes, but did not, unlike venlafaxine, have any side-effects. Rather the women treated with acupuncture recorded positive effects such as increased energy levels, clarity of thought and an increased sex drive. 40

Studies investigating the effect of acupuncture in the treatment of menopausal hot flashes in healthy women have generally been small and have reported mixed results, although a recent randomized study from Norway $(\mathrm{n}=267)$ demonstrated a significant hot flash reduction in women receiving acupuncture and self-care compared to those only receiving self-care. ${ }^{41}$ The acupuncture group also showed significant improvements in sleep and somatic symptoms. Similarly, studies investigating acupuncture as a treatment for hot flashes in breast cancer patients have been small pilot studies. ${ }^{42-46}$ However, the last few years have seen an increase in randomized, controlled studies in this field. The authors of this paper have previously published a controlled randomized trial $(\mathrm{n}=59)$ demonstrating that acupuncture has a significant effect on the reduction of hot flashes and an increased health related quality of life for up to three months post-treatment (Table 2).30 Frisk demonstrated a reduction in hot flashes for up to 24 months after electro-acupuncture treatment $(n=45)$ compared with HRT.47 Walker, in a randomized, controlled study $(\mathrm{n}=50)$ comparing acupuncture to venlafaxine, found that the effects of acupuncture lasted for two months post treatment compared to two weeks

Table 1. Comparing the side effects of aromatase inhibitors to tamoxifen.

\begin{tabular}{|c|c|c|c|c|c|c|c|c|c|c|}
\hline $\begin{array}{l}\text { Side } \\
\text { effects }\end{array}$ & $\begin{array}{l}\text { Hot } \\
\text { flashes }\end{array}$ & $\begin{array}{l}\text { Weight } \\
\text { gain }\end{array}$ & Insomnia & $\begin{array}{l}\text { Joint } \\
\text { aches }\end{array}$ & $\begin{array}{l}\text { Loss of } \\
\text { libido }\end{array}$ & $\begin{array}{l}\text { Mood } \\
\text { changes }\end{array}$ & $\begin{array}{l}\text { Muscle } \\
\text { aches }\end{array}$ & $\begin{array}{l}\text { Vaginal } \\
\text { dryness }\end{array}$ & $\begin{array}{l}\text { Vaginal } \\
\text { discharge }\end{array}$ & $\begin{array}{c}\text { Severe } \\
\text { osteo-porosis }\end{array}$ \\
\hline TAM & $35 \%$ & $14 \%$ & $17 \%$ & $12 \%$ & $79 \%$ & $81 \%$ & $84 \%$ & $75 \%$ & $80 \%$ & $2.9 \%$ \\
\hline $\mathrm{AI}$ & $30 \%$ & $15 \%$ & $18 \%$ & $22 \%$ & $77 \%$ & $76 \%$ & $84 \%$ & $70 \%$ & $71.9 \%$ & $5.7 \%$ \\
\hline
\end{tabular}

TAM, tamoxifen; AI, aromatase inhibitor. 
in the women medicated with venlafaxine. ${ }^{40} \mathrm{In}$ a one arm study $(\mathrm{n}=50)$, De Valois et al. demonstrated a reduction of hot flashes at 18 weeks post treatment. 48 However, two recently published randomized, controlled studies from the USA and Sweden could not demonstrate that acupuncture was more effective than sham in reducing vasomotor symptoms. ${ }^{49-50}$

Menopause in healthy women in TCM is regarded as a natural transition. Menopausal symptoms are considered to be a decline of kidney essence alone, or combined with excesses such as stasis of qi and blood, dampness and heat (empty and full). During the woman's $7^{\text {th }}$ cycle, kidney qi diminishes, the ren and chong channels have no nourishment, periods become unpredictable and eventually stop. Kidney qi decline may upset the balance of yin and yang, the body temperature is poorly regulated, usually a deficiency of kidney yin prevails, and empty heat gives rise to hot flashes. Symptoms due to anti-estrogen medication in ER+ breast cancer patients are similar to those in healthy menopausal women, the main symptoms being hot flashes, tiredness, body aches and pains, insomnia and emotional imbalance. However, symptoms are often more severe in breast cancer patients. ${ }^{51}$ Though the most likely syndrome in women suffering from hot flashes due to side-effects of anti-estrogen medication is a kidney yin deficiency, a yang deficiency may also be present, though to a lesser extent. Liver yin deficiency is often seen, especially in patients who also complain of sore, stiff joints. Symptoms for these syndromes are similar, but a closer look may reveal separate disharmonies. The underlying full patterns related to the original diagnosis, including stagnation of qi, damp, blood and the accumulation of phlegm if still present would first need to be addressed. One source of classical medical literature from 1637 states whenever we treat a disease, we must approach it at the base. 52

\section{Insomnia}

Numerous studies have shown that insomnia, fatigue and hot flashes affect each other. In a study investigating the effect of acupuncture for the treatment of hot flashes in breast cancer patients, a combination of hot flashes, sleep disturbances and fatigue found at base line were similarly improved following treatment. ${ }^{30}$ In healthy postmenopausal women, objectively recorded hot flashes and recorded waking episodes are associated with sleep disruption. ${ }^{3}$ Sleep difficulties are reported by 30 $50 \%$ of newly diagnosed cancer patients. ${ }^{54}$ Breast cancer is associated with a particularly high rate of insomnia compared to other cancer types; prevalence estimates vary from $38 \%$ to $61 \% .55$ Both psychological and physical factors related to breast cancer probably contribute to sleep problems. Hot flashes occur- ring at night have been reported as being more severe and bothersome than daytime episodes, causing sleep disturbance. 56 In a study examining the relationship between objectively measured nocturnal hot flashes and objectively measured sleep in breast cancer survivors with insomnia $(\mathrm{n}=24)$, Savard et al. found that during the 10-minute periods around hot flashes, participants were, on average, in the wake stage for a higher percentage of time (31\%) compared to non-hot-flash 10-minute periods (19\%). ${ }^{57}$ This suggests that the mechanism for sleep disruption is coincident with that of hot flashes, leading to both an arousal from sleep and vasomotor response. Arousals were found not to be in response to the discomfort due to the hot flashes, because the arousals generally precede vasomotor response (Table 3).

TCM views the heart as the main organ associated with consciousness, intelligence and sleep. The heart is the root of life, the seat of Shen, the master of blood and the commander of the vessels. An important function of the heart is the storing of Shen, the spirit. When this function is impaired, due to disharmony of blood and qi, Shen is not nourished, symptoms such as insomnia, restlessness, excessive dreaming and mental fogginess may occur. In extreme cases, serious psychological problems can arise. There is a close relationship between the qi of the chest (zhong qi) and the heart. Zhong qi helps the lungs with the movement of inspiration and expiration thath in turn helps to move qi, and helps the heart manage the heart beat and movement of blood. All these functions in the chest depend on each other and any inbalance can have far reaching consequences. Heart blood and qi are dependent on each other; any unbalance will affect the heart beat and, therefore, the pulse.
Emotional problems affecting the chest area are grief and sadness. These emotions are often encountered in middle aged to older women and are undoubtedly exaggerated by breast cancer diagnosis. Insomnia is usually related to disturbances of the heart storing Shen, but may be connected to other organs as well. Typically, women with breast cancer complain of symptoms such as insomnia, fatigue, palpitations, anxiety and cognitive fogginess. Insomnia is often associated with hot flashes. When questioned closely, most women complain of heat around the head and neck, the chest and the palms of their hands, all signs of empty heat. Insomnia combined with symptoms of empty heat and mental restlessness usually indicates a pattern of kidney/heart disharmony inbalance. Inspection of the tongue typically reveals a red tip, indicating emotional stress, stagnation of qi and hence heat affecting the heart. The pulse will typically be rapid and thin, and possibly deep and weak in the two-third positions if there is a degree of yang xu. The complexion is an important indicator of the state of the heart and the blood. If the heart and the blood are harmonized, the complexion is healthy, glossy and rosy; if deficient the complexion will be pale and dull. The aim of acupuncture treatment would be to tonify kidney and heart yin, reduce heat and calm the mind. In The Yellow Emperor's Classic of Medicine, Neijing states that shen is the light that shines out of the eyes. ${ }^{58}$ Bright, lively eyes indicate that Shen is healthy, the balance between sleep and awake time is good, and the person is mentally alert. The heart is connected to the kidney. Neijing further states that the heart resides in the vessels. It rules the kidney network because it is the general master of all organ networks.

Table 2. Comparison of mean number of hot flashes before and after 15 acupuncture treatments, and 3 months post treatment.

\begin{tabular}{lccc} 
& Baseline & Last treatment & 3 months post treatment \\
Night & $6 / 7.2$ & $2.6 / 5.4$ & $1.7 / 6.1$ \\
$\begin{array}{l}\text { Mean number HF } \\
\text { Acupuncture/sham }\end{array}$ & & & \\
$\begin{array}{l}\text { Day } \\
\text { Mean number HF } \\
\text { Acupuncture/sham }\end{array}$ & $9.5 / 12.3$ & $4.7 / 11.7$ & $3.1 / 12.1$ \\
\hline
\end{tabular}

$\mathrm{HF}$, hot flashes.

Table 3. Mean sleep disruption around 10 minute hot flash and non hot flash periods. 10 minute periods around HF Other 10 minute periods

\begin{tabular}{lcc} 
Number of awakenings & 0.38 & 0.59 \\
Percent wake time & 31.22 & 18.93 \\
\hline
\end{tabular}

\begin{tabular}{lll}
\hline Number of stage changes to lighter sleep & 1.61 & 1.20
\end{tabular}

HF, hot flashes. 
Before the heart fire can harmoniously blend with the kidney water, however, the kidney water must be sufficient. Otherwise the heart fire will flare out of control, and all kinds of heart and kidney ailments will arise.

A review of controlled studies published in the Cochrane Central Register before 2007 revealed that studies investigating the effect of acupuncture on patients complaining of insomnia were few, with poor methodology and significant clinical heterogeneity. The studies reviewed did not, therefore, provide sufficient evidence to support the use of acupuncture for the treatment of insomnia. ${ }^{59}$ However, it may be hypothesized that since the central and autonomic nervous systems are considered to be involved in sleep dysfunction, by its effect on peripheral nerves, modulating autonomic activity and central activation, acupuncture could potentially influence a number of neural and hormonal mechanisms affecting sleep regulation.

\section{Muscle and joint pains}

Patients taking both TAM and AIs complain of joint (TAM 12\%, AI 22\%) and muscle (TAM 84\%, AI 84\%) aching. ${ }^{23}$ Those taking AIs report more severe symptoms which are the reason for $27 \%$ of women stopping taking this type of drug; 5 a decision that could have dire consequences. Women taking AIs are advised to take regular bone density tests. Estrogen has a protective effect on bone via multiple mechanisms; in short, it stimulates new bone formation and inhibits bone reabsorption. ${ }^{60}$ In estrogen deficiency states, bone reabsorption predominates and bone loss ensues. AIs cause bone loss by lowering the levels of endogenous estrogen. In contrast, TAM has tissue-specific estrogen agonist effects. In bone of postmenopausal women, TAM acts as a weak estrogen to preserve bone mineral density and may decrease fractures. 61,62

TCM considers the bones to be under the influence of the kidneys and the tendons to be the responsibility of the liver. The kidneys are said to rule the bones, producing bone marrow from jing. Failure of the kidneys to rule the bones due to deficiency will lead to osteoporosis. Deficiency of the liver can affect the tendons that rely on liver blood for nourishment so that they can facilitate smooth movement. Blood stasis, caused by stagnation of qi and blood in the channels, causes pain. Women medicated with AIs complain of dull aches in their joints, which are also stiff, especially after rest, and stabbing pains related to muscles. ${ }^{63} \mathrm{~A}$ dull ache is by its nature a type of deficiency; stiffness is characterized by the tendons not being nourished by blood, stabbing pains are a sign of blood stagnation. A mixture of deficiency and excess emerges. Since women taking Als experience more severe joint and muscle pain, and an increased risk of developing osteoporosis, it could be suggested that the level of kidney and liver deficiency, and, therefore, qi and blood stagnation, is more severe than in those women medicated with TAM. Indeed, aromatase inhibitors are considered to be slightly superior to TAM, due to better disease free survival rates after 5 years, $84.0 \%$ for letrozole (AI) and $81.4 \%$ for TAM. ${ }^{4}$ Trials examining the quality of life at various time points after these women have stopped taking their 5 year course of antiestrogen medication are sadly lacking.

The authors were unable to find any trials concerning the treatment of muscle and joint pain with acupuncture specifically in breast cancer patients. However, the use of acupuncture in reducing musculoskeletal pain has been an area of interest in the West since the early 1970s. Accepted mechanisms involved in pain reduction and control in response to acupuncture include the systemic release of adrenocorticotropic hormone and $\beta$-endorphins, potentially reducing pain and inflammation. 65 An article examining eight Cochrane reviews concerning pain syndromes concluded that acupuncture is effective for migraines, neck disorders, tension-type headaches and peripheral joint osteoarthritis. 66

\section{Emotional issues and reduced sex drive}

TCM considers emotional issues to be the most important cause of breast cancer. Blood stasis, or phlegm due often to spleen deficiency causing dampness, frequently a consequence of qi stagnation, is often brought on by worry, sadness, frustration or anger. Although stagnation of qi is usually connected to the liver, emotions can affect qi in the organs of the chest area, the heart and lungs. The lung channel flows laterally to the breasts, stagnation of lung qi could therefore lead to lumps in the chest or breasts. Typical emotional causes of weakened qi that does not flow properly and eventually becomes stagnant are: worry affecting the spleen, sadness affecting the lungs, frustration or anger affecting the liver, fear affecting the kidneys and unhappiness affecting the heart. A recent study from Israel demonstrated that happiness and optimism may play a preventative role in breast cancer, while adverse life events can increase the risk of developing the disease. $67 \mathrm{~A}$ total of 622 women between the ages of 25 and 45 were interviewed and results showed a clear link between outlook and risk of breast cancer, with optimists $25 \%$ less likely to develop the disease. Conversely, women who suffered two or more traumatic events had a $62 \%$ greater risk of developing breast cancer. A possible highrisk group for breast cancer could be identified made up of young women who had been exposed to a number of negative life events. Associations between stress and cancer were further confirmed in a review of 165 studies indicating that stress-related psychosocial factors are associated with higher cancer incidence in initially healthy populations. ${ }^{68}$ An area of worry for many women medicated with estrogen antagonists is mental fogginess that may be due to a number of inbalances, including phlegm clouding the mind, kidney xu not nourishing the brain, or empty heat due to kidney yin xu harassing the mind. Forty-six percent of the AI medicated women answering a survey conducted by Breast Cancer Action $(n=1,199)$ reported experiencing mental fuzziness (possibly early signs of the loss of some cognitive functions). ${ }^{15}$

Around 75\% of women medicated with estrogen antagonists complain of reduced sex drive. More than $70 \%$ of women taking TAM and AIs report vaginal dryness and discomfort.23 In a qualitative study carried out by the authors of this paper, women complained of not feeling attractive, reduced libido and vaginal problems. ${ }^{63}$ One may hypothesize that middle aged to older women have many emotional issues to cope with; ageing, feeling less physically attractive, children leaving home, divorce, and ageing or death of parents. Any one or a few of these issues, together with a fall in sex hormones, can possibly lead to emotional distress and reduced libido. In breast cancer, the breast, that is such an important emotional link, a source of nourishment and comfort to children, important in the definition of the female shape and sexuality, becomes diseased. Furthermore, especially when a mastectomy is carried out, breast surgery may have a deep affect on women who may mourn for their lost breast, their femininity and sexuality. This may cause new emotional problems to arise affecting an already unstable system.

Although the authors could not find any studies on acupuncture that primarily addressed sex drive and well being, these parameters are often included in quality of life questionnaires. In several studies, acupuncture has been shown to have a positive effect on well-being in breast cancer patients, ${ }^{48,30}$ and in a study investigating the effect of acupuncture in 50 anti-estrogen medicated women, $25 \%$ reported that their sex-drive had increased. ${ }^{40}$ Acupuncture is a popular treatment for depression and anxiety. Manual acupuncture has been shown to elicit a range of central nervous system responses involving the amygdala, hippocampus, hypothalamus, cerebellum and other limbic structures seen on functional magnetic resonance imaging and electroencephalogram. ${ }^{69}$ In a study comparing acupuncture and Prozac in 440 patients, the therapeutic effect of acupuncture on depressive neurosis was shown to be significantly better than that of prozac and to have fewer side-effects. ${ }^{70}$ 


\section{Conclusions}

Treatment of ER+ breast cancer tumors with estrogen antagonist therapy is constantly being reassessed. TAM has traditionally been the initial drug of choice in both pre- and postmenopausal women. However, aromatase inhibitors increasingly represent the treatment of choice in post-menopausal women. Aromatase inhibitors have proved to be superior to TAM both in reducing early recurrence, and improving long-term survival rates. Although the side-effects of these drug types are similar, there are also some differences. Little scientific research has investigated the effect of TCM in the treatment of side-effects related to these drugs. Most research has been concentrated on the main side-effect of both drugs, i.e. hot flashes. However, with the increasing use of aromatase inhibitors, joint and muscle pains, the main reason women give for stopping this medication, should be a focus. Numerous studies have demonstrated the role of acupuncture as a supportive treatment in the post-operative phase in this patient group, advantages include the absence of clinically relevant side-effects and the low cost of treatment. Breast cancer in the East is currently treated with conventional medicine and TCM is used to support treatment, treat side-effects and restore health. Patients in the West are increasingly interested in this marriage of Eastern and Western medical systems. Integrative medicine, a system of mutual support between complementary and alternative medical (CAM) therapies and conventional medicine is increasingly popular in America and is also attracting attention in Europe. The validity of TCM as a treatment option for women with side-effects from adjuvant breast cancer treatment is dependent upon clinically relevant, well designed randomized, controlled studies with sufficient statistical power. Few medical institutions in Europe offer TCM as a standard treatment. University departments offering research positions in complementary and alternative medicine are often small, funding for clinical trials in such areas is scarce, and research supervision is typically lacking. Thus, CAM research often loses out to conventional medicine that is supported by educational institutions and powerful drug companies in the fight for funding. Without large randomized trials, TCM will always be the little sister in the West.

\section{References}

1. Boyle P. Levin B. World Cancer Report. Lyon: IARC Press; 2008.

2. International Agency for Research on
Cancer. Globocan Cancer Fact sheets GLOBOCAN 2008. Available from: globocan.iarc.fr/factsheets/cancers/all.asp. Accessed on: 15 July 2012.

3. Perry N, Broeders M, de Wolf $\mathrm{C}$, et al. European guidelines for quality assurance in breast cancer screening and diagnosis, fourth edition-summary document. Ann Oncol 2008;19:614-22.

4. Langseth H, Gislefoss RE, Martinsen JI, et al. Cancer Registry of Norway, Cancer in Norway 2008. Cancer incidence, mortality, survival and prevalence in Norway. Oslo: Cancer Registry of Norway; 2009.

5. Early Breast Cancer Trialists' Collaberative group. TAM for early breast cancer, an overview of the randomized trials. Lancet 1998;351:1451-67.

6. Thurlimann B, Robertson JF, Nabholtz JM, et al. Efficacy of TAM following anastrozole (Arimidex') compared with anastrozole following TAM as first-line treatment for advanced breast cancer in postmenopausal women. Eur J Cancer 2003; 39:2310-7.

7. Bonneterre J, Buzdar A, Nabholtz JM, et al. Anastrozole is superior to TAM as first-line therapy in hormone receptor positive advanced breast carcinoma. Cancer 2001; 92:2247-58.

8. Garrow JR, Delamelena T, Walts D. et al. Side effects of aromatase inhibitors versus TAM: the patients' perspective. Am J Surg 2006;192:469-98.

9. Maciocia G. Obstetrics and gynecology in Chinese medicine. New York, USA: Churchill Livingstone; 1998.

10. Jin GY, Lue FL. Discussion of prescriptions of the golden chest. Zhejiang: Zhejiang Scientific Publishing House; 1981.

11. The Yellow emperor's classic of internal medicine. Simple questions (Huang Di Nei Su Wen). Beijing: People's Health Publishing House; 1979.

12. Flaws B, Yang SZ. Master Hua's classic of the central viscera: at of the Zhong Zang Jing. Boulder, CO: Blue poppy press; 1993.

13. Cohen I, Tagliaferri M, Tripathy D. Traditional Chinese Medicine in the treatment of breast cancer. Semin Oncol 2002; 29:563-74.

14. Dundee JW, Yang J, Mcmillan C. Noninvasive stimulation of P6 (Neiguan) antiemetic acupuncture point in cancer chemotherapy. J R Soc Med 1991;84:210-2.

15. Zivian M, Salgado B. Breast cancer action group: side-effects revisited: women's experiences with aromatase inhibitors. 2008. Available from: www.bcaction.org.

16. Hersham DL, Kushi LH, Shao T, et al. Early discontinuation and nonadherence to adjuvant hormonal therapy in a cohort of 8,769 early-stage breast cancer patients. J Clin Oncol 2010;28:4120-8.
17. The Italian group for antiemetic research. Dexamethasone, granisteron, or both for the prevention of nausea and vomiting during chemotherapy for cancer. N Engl J Med 1995;332:1-5.

18. Dundee JW, Chestnut WN, Ghaly RG et al. Traditional Chinese acupuncture: a potentially useful antiemetic? Br Med J 1986; 293:583-4.

19. Gan TJ, Jiao KR, Zenn M, Georgeade GA. A randomized controlled comparison of electro-acupoint stimulation or ondansetron versus placebo for the prevention of postoperative nausea and vomiting. Anesth Analg 2004;99:1070-5.

20. Han YF, Gong Z, Huang LQ, et al. Clinical study on acupuncture for leukopenia induced by chemotherapy. Zhongguo Zhen Jiu 2010;30:802-5. [Article in Chinese].

21. Worsham MJ, Raju U, Lu M, et al. Risk factors for breast cancer from benign breast disease in a diverse population. Breast Cancer Res Treat 2009;118:1-7.

22. Lahans T. Treatment principles in Chinese medicine for modern integrated cancer care. Churchill Livingstone, Elsevier, 2007

23. Sestak I, Cuzick J, Sapunar F, et al. ATAC Trialists' Group Risk factors for joint symptoms in patients enrolled in the ATAC trial: a retrospective, exploratory analysis. Lancet Oncol 2008;9:866-72.

24. National Institutes of Health State-of-theScience Panel. National Institutes of Health State-of-the-Science Conference statement: management of menopauserelated symptoms. Ann Intern Med 2005;142: 1003-13.

25. Coleman RE, Bolten WW, Lansdown M. Aromatase inhibitor-induced arthralgia: clinical experience and treatment recommendations. Cancer Treat Rev 2008;34: 275-82.

26. Kronenberg F. Hot flashes phenomenology, quality of life, and search for treatment options. Exp Gerontol 1994;29:319-36.

27. Love RR, Cameron L, Connell BL, Levanthal H. Symptoms associated with TAM treatment in postmenopausal women. Arch Intern Med 1991;151:1842-7.

28. Ilnyckyj A, Shanahan F, Anton PA, et al. Quantification of the placebo response in ulcerative colitis. Gastroenterology 1997;112:1854-8.

29. Miller HG, Li RM. Measuring hot flashes: summary of a national institutes of health workshop. Mayo Clin Proc 2004;79:668-70.

30. Hervik $\mathrm{J}$, Mjåland 0 . Acupuncture for the treatment of hot flashes in breast cancer patients, a randomized, controlled trial. Breast Cancer Res Treat 2009;116:311-6.

31. Mohyi D, Tabassi K, Simon J. Differential diagnosis of hot flashes. Maturitas 1997;27:203-14.

32. Stearns V, Hayes DF. Cooling off hot flash- 
es. J Clin Oncol 2002;20:1436-8.

33. Kouriefs C, Georgiou M, Ravi R. Hot flushes and prostate cancer: pathogenesis and treatment. BJU International 2002;89:37983.

34. Freedman RR, Krell W. Reduced thermoregulatory null zone in postmenopausal women with hot flashes. Am J Obstet Gynecol 1999;181:66-70.

35. Stearns V, Ullmer L, López JF, et al. Hot flushes. Lancet 2002;360:1851-61

36. Hickey M, Saunders C, Partridge A, et al. Practical clinical guidelines for assessing and managing menopausal symptoms after breast cancer. Ann Oncol 2008; 19:1669-80.

37. Stearns V, Johnson MD, Rae JM, et al. Active TAM metabolite plasma concentrations after coadministration of TAM and the selective reuptake inhibitor paroxetine. J Nat Cancer Inst 2003;95:1758-64.

38. Goetz MP, Knox SK, Suman VJ, et al. The impact of cytochrome P450 2D6 metabolism in women receiving adjuvant TAM. Breast Cancer Res Treat 2007;101:113-21.

39. Osborne CK. TAM in the treatment of breast cancer. N Engl Med 1998;339:160918.

40. Walker EM, Rodriguez AI, Kohn B, et al. Acupuncture versus Venlafaxine for the management of vasomotor symptoms in patients with hormone receptor-positive breast cancer: a randomized controlled trial. J Clin Oncol 2010;28:634-40.

41. Borud EK, Alraek T, White A, Grimstad S. The acupuncture on hot flushes among menopausal women (ACUFLASH) study: observational follow up results at six months. Menopause 2010;17:262-8.

42. Filshie J, Bolton T, Browne D, Ashley S. Acupuncture and self acupuncture for long- term treatment of vasomotor symptoms in cancer patients-audit and treatment algorithm. Acupunct Med 2006;23: 171-80.

43. Nedstrand E, Wijma K, Wyon Y, Hammar M. Vasomotor symptoms decrease in women with breast cancer randomised to treatment with applied relaxation or electro-acupuncture: a preliminary study. Climacteric 2005;8:243-50.

44. Tukmachi E. Treatment of hot flushes in breast cancer patients with acupuncture. Acupunct Med 2000;18:2-7.

45. Porzio G, Trapasso CT, Martelli S, et al. Acupuncture in the treatment of vasomo- tor symptoms in postmenopausal women taking TAM. Tumori 2002;88:128-30.

46. Towlerton G, Filshie J, O Brien M, Duncan A. Acupuncture in the control of vasomotor symptoms caused by TAM. Palliat Med 1999;13:445.

47. Frisk J, Carlhåll S, Kålstrøm AC, et al. A long term follow-up of acupuncture and hormone therapy on hot flushes in women with breast cancer: a prospective, randomized, controlled, multicentre trial. Climacteric 2008;11:166-74.

48. De Valois, Young TE, Robinson N, et al. Using traditional acupuncture for breast cancer-related hot flashes and night sweats. Altern Complement Med 2010; 16:1047-57.

49. Deng G, Vickers A, Yeung S, Cassileth B. Randomised, controlled trial of acupuncture for the treatment of hot flashes in breast cancer patients. J Clin Oncol 2007;35:5584-90.

50. Liljegren A, Gunnarsson P, Landgren BM, et al. Reducing vasomotor symptoms with acupuncture in breast cancer patients treated with adjuvant TAM: a randomized controlled trial. Breast Cancer Res Treat 2010 [Epub ahead of print].

51. Boekhout, AH. Beijnen, JH. Schellens, JH, Symptoms and treatment of cancer therapy-induced early menopause. Onchologist 2006;11:641-54.

52. Li Zhongzi. A primer of medical objectives (Yizong Bidu), 1637.

53. Gonen R, Sharf M, Lavie P. The association between mid-sleep waking episodes and hot flushes in post-menopausal women. J Psychosom Obst Gynaecol 1986;5:113-7.

54. Savard J, Morin CM. Insomnia in the context of cancer: a review of a neglected problem. J Clin Oncol 2001;19:895-908.

55. Savard J, Simard S, Blanchet J, et al. Prevalence, clinical characteristics, and risk factors for insomnia in the context of breast cancer. Sleep 2001;24:583-89.

56. Carpenter JS, Johnson D, Wagner L, et al. Hot flashes and related outcomes in breast cancer survivors and matched comparison women. ONF 2002;29:E16-25.

57. Savard J, Davidsen JR, Ivers H, et al. The association between nocturnal hot flashes and sleep in breast cancer survivors. $\mathrm{J}$ Pain Symptom Manage 2004;27:513-22.

58. The Yellow Emperor's classic of medicine (the Neijing Suwen). Shambala Publications; 1995.
59. Cheuk DK, Yeung WF, Chung KF, Wong V. Acupuncture for insomnia. Cochrane Database Syst Rev 2007;18:CD005472.

60. Ramaswamy B, Shapiro CL. Osteopenia and osteoporosis in women with breast cancer. Semin Oncol 2003;30:763-75.

61. Love RR, Mazess RB, Barden HS, et al: Effects of TAM on bone mineral density in postmenopausal women with breast cancer. N Engl J Med 1992;326:852-6.

62. Fisher B, Costantino JP, Wickerham DL, et al: TAM for prevention of breast cancer: report of the national surgical adjuvant breast and bowel project P-1 study. J Natl Cancer Inst 1998;90:1371-88.

63. Hervik $\mathrm{J}$, Mjåland 0 . Quality of life of breast cancer patients medicated with anti-estrogens, two years after acupuncture treatment. A qualitative study. Int $\mathrm{J}$ Womens Health 2010;28:319-25.

64. Giobbie-Hurder A, Price KN, Gelber RD. International Breast Cancer Study Group; BIG 1-98 Collaborative Group Design, conduct, and analyses of Breast International Group (BIG) 1-98: a randomized, doubleblind, phase-III study comparing letrozole and TAM as adjuvant endocrine therapy for postmenopausal women with receptorpositive, early breast cancer. Clin Trials 2009;6:272-87.

65. Li A, Lao L, Wang Y, et al. Electroacupuncture activates corticotrophin-releasing hormone-containing neurons in the paraventricular nucleus of the hypothalamus to alleviate edema in a rat model of inflammation. BMC Complement Altern Med 2008;8:20.

66. Lee MS, Ernst E. Acupuncture for pain: an overview of cochrane reviews. Chin J Integr Med 2011;17:187-9.

67. Peled R, Carmil D, Siboni-Samocha 0, Shoham-Vardi I. Breast cancer, psychological distress and life events among young women. BMC Cancer 2008;8:245.

68. Chida Y, Hamer M, Wardle J, Steptoe A. Do stress-related psychosocial factors contribute to cancer incidence and survival? Nat Clin Pract Oncol 2008;5:466-75.

69. Hui KK, Napadow V, Liu J, et al. Monitoring acupuncture effects on human brain by FMRI. J Vis Exp 2010;8:1190.

70. Fu WB, Fan L, Zhu XP, et al. Depressive neurosis treated by acupuncture for regulating the liver-a report of 176 cases. Tradit Chin Med 2009;29:83-6. 